$\Rightarrow$ DIABETES

\title{
Renal responses to SGLT2 blockade
}

\section{cef \\ Hyperketonaemia induced by SGLT2 blockade results from enhanced ketogenesis}

Inhibition of the sodium-glucose cotransporter 2 (SLGT2) efficiently controls glycaemia in patients with type 2 diabetes mellitus (T2DM) but it can also induce ketoacidosis. In a new study, Ele Ferrannini and colleagues show that the hyperketonaemia induced by SGLT2 blockade results from enhanced ketogenesis and not from reduced ketonuria.

Previous work from the researchers showed that SGLT2 inhibition in patients with T2DM increases circulating ketone levels. "As little is known about the renal handling of ketones outside of prolonged fast, we tested whether the increase in plasma $\beta$-hydroxybutyrate $(\beta$-HB) - the main ketone body - might be due to changes in its renal clearance," explains Ferrannini. The researchers measured excretion rates of glucose, sodium, $\beta-\mathrm{HB}$ and lactate in the urine of 66 patients with T2DM and preserved renal function and in 25 nondiabetic individuals with normal $(n=12)$ or impaired glucose tolerance $(n=13)$, following acute or chronic SGLT2 inhibition with empagliflozin.

Chronic (4 weeks) or acute (single dose) administration of empagliflozin increased excretion of $\beta-\mathrm{HB}$, sodium and lactate in all individuals; excretion correlated with glycosuria and was independent of BMI and creatinine clearance. "Glycosuria, with the attendant osmotic diuresis, might exert a 'dragging' effect on filtered solutes,' says Ferrannini. SGLT2 blockade did not alter levels of pro-B-type natriuretic peptide but increased plasma levels of erythropoietin. "The glycosuria and natriuresis caused by SGLT2 blockade might have unsuspected consequences on renal metabolism and function, such as cause an intrarenal shift in sodium handling, changes in the excretion of other metabolites and proteins and in the substrate mix used for energy production," explains Ferrannini. The researchers suspect such changes might explain the renoprotective effects of chronic SGLT2 inhibition, which could extend use of these agents to non-diabetic nephropathies.

Andrea Aguilar

ORIGINAL ARTICLE Ferrannini, E. et al. Renal handling of ketones in response to sodium-glucose cotransporter 2 inhibition in patients with type 2 diabetes. Diabetes Care. http://dx.doi.org/10.2337/dc16-2724 (2017) 